\title{
AvanCES EN EL ESTUdio DE CERÁMICA y PIGMENTOS EN ARQUEOLOGÍA: UNA INTRODUCCIÓN
}

Advances in ceramic and pigment analysis in Archaeology: An introduction

\author{
Jalh Dulanto a \\ Isabelle Druc ${ }^{b}$
}

El valor de estos dos volúmenes, que son publicados como los números 26 y 27 del Boletín de Arqueología PUCP, radica sin dudas en las descripciones detalladas de una amplia variedad de metodologías utilizadas en una amplia variedad de casos de estudio de vasijas de cerámica y pigmentos arqueológicos de Perú, Chile y Argentina. Juntos nos brindan una imagen de los avances en los métodos y técnicas aplicados al análisis de estos materiales —en especial, el uso de XRF, espectroscopia Raman, microscopía digital portátil en luz reflexiva, y Quemscan-. Pero, también, de la persistencia de métodos y técnicas clásicos, cuya confiabilidad y validez para mejorar nuestra comprensión de la producción, intercambio y consumo de objetos de cerámica ha sido comprobada en múltiples oportunidades, principalmente, la petrografía, que permite la caracterización de la tecnología cerámica, la difracción de rayos X que permite la identificación precisa de muchos de los minerales presentes en una muestra cristalina y en particular en muestras de arcillas no quemadas, y el análisis de activación de neutrones, que permite conocer en detalle la composición química de los materiales estudiados. El valor de combinar de manera creativa e innovadora estos métodos y técnicas, nuevos y viejos, es resaltado en muchos de los artículos incluidos en estos dos volúmenes.

Especial atención, merece el énfasis puesto por varios de los autores en la importancia de conocer la variedad de los materiales estudiados no solo en una escala local, sino también regional, y sobre todo de conocerla a partir de un diálogo constante entre especialistas pertenecientes a múltiples disciplinas, que incluyen no solo a las tradicionalmente vinculadas a las ciencias humanas, como la arqueología, la historia y la etnografía, sino también a las tradicionalmente vinculadas a las ciencias naturales, como la geología, la mineralogía y la química. La necesidad de cruzar disciplinas y relacionar a especialistas de estos dos ámbitos es, en ese sentido, ineludible. Estos dos volúmenes reúnen a arqueólogos, historiadores del arte, etnógrafos, químicos, físicos, geólogos, e ingenieros.

Abrimos este primer volumen con un artículo de Kusi Colonna-Preti, Peter Eeckhout y Milton Luján sobre los murales recientemente descubiertos en el edificio B15 de Pachacamac. Los autores

\footnotetext{
a Sección de Arqueología, Pontificia Universidad Católica del Perú Correo electrónico: jdulant@pucp.pe

b Department of Anthropology, University of Wisconsin-Madison

Correo electrónico: icdruc@wisc.edu
} 
presentan los resultados de un estudio detallado de la microestratigrafía de los murales, los materiales utilizados en su elaboración y los instrumentos usados para pintarlos, que les permiten concluir que la mayoría de ellos fueron elaborados con pinturas locales, aunque posiblemente también importadas, que fueron mezcladas en el lugar, y aplicadas sobre los muros, para renovar periódicamente los espacios que decoraban siguiendo patrones y regularidades que aún están siendo investigados, pero que muy probablemente son una función del estado de conservación de los mismos.

Nino Del Solar, Rémy Chapoulie y Luis Jaime Castillo presentan los resultados de su estudio de la composición mineralógica y química de las pinturas utilizadas en vasijas de cerámica de los estilos mochica, cajamarca costeńo y cajamarca serrano del sitio arqueológico de San José de Moro en el valle de Jequetepeque. Los análisis de fluorescencia de rayos X portátil (pXRF) y espectrometría Raman sugieren interesantes cambios en las soluciones técnicas utilizadas en la decoración de estas vasijas a partir de la influencia huari en la región y contribuyen a nuestro entendimiento de la interacción entre poblaciones costeńas y serranas en el norte del Perú.

Isabelle Druc, Silvana Bertolino, Andrée Valley, Kinya Inokuchi, Francisco Rumiche y John Fournelle analizan el caso de las vasijas del estilo Cupisnique Rojo Grafitado del primer milenio a.C. de la costa norte del Perú. El análisis que combina varios métodos y técnicas arqueométricos les permite determinar que los alfareros que produjeron estas vasijas compartían las mismas técnicas pero no los recursos y que en Kuntur Wasi, el famoso centro ceremonial de este período, ubicado en la sierra adyacente, este estilo fue imitado en nuevas variantes morfológicas y decorativas.

Jalh Dulanto, Patricia Gonzáles y Enrique Guadalupe presentan los resultados del estudio de los pigmentos utilizados en la pintura poscocción de vasijas de cerámica Paracas Temprano de Puerto Nuevo, que les permiten hacer una serie de inferencias acerca de las redes de intercambio de la costa y sierra surcentral del Perú, durante los siglos IX-VI A.C. A partir del análisis por fluorescencia de rayos X portátil (pXRF), espectrometría Raman, difracción de rayos X (DRX) y microespectroscopía infrarroja transformada de Fourier (micro-FTIR), logran identificar los principales minerales utilizados en la elaboración de pigmentos de diferentes colores. El análisis de la distribución de yacimientos de estos minerales les permite concluir que durante el período en cuestión las poblaciones de la costa, no solo obtuvieron pigmentos procedentes en su entorno inmediato, sino también pigmentos procedentes de la sierra adyacente.

Alicia Espinosa, Gabriel Prieto, Walter Alva y Claude Chauchat presentan un estudio de tecnología cerámicas para identificar tradiciones técnicas vinculadas a diferentes culturas arqueológicas. A partir de un análisis detallado de huellas macroscópicas y microscópicas logran identificar tres tradiciones técnicas vinculadas a dos comunidades identificadas con estas culturas: la tradición de martillado vinculada a comunidades Virú-Gallinazo y las tradiciones de enrollado y moldeado vinculadas a comunidades mochica. Este hallazgo los lleva a cuestionar las descripciones tipológicas y estilísticas como indicadores fiables de filiación cultural. Su estudio es una clara prueba de que no es necesario recurrir a la última técnica arqueométrica para llegar a conclusiones interesantes sobre prácticas del pasado.

Vanesa Juarez y Beatriz Cremonte presentan los resultados de sus estudios de pastas de vasijas de cerámica del sitio arqueológico de Las Garzas del valle de Lerma en Salta, Argentina. El análisis petrográfico de las pastas de estas vasijas y de arcillas locales les permiten acercarse a la identificación de las fuentes de arcilla explotadas para la elaboración de las vasijas, mientras que la comparación de las mismas con pastas de vasijas procedentes de otros sitios arqueológicos del mismo valle, y de los valles más distantes de Tafí y San Francisco, les permite establecer nexos en las técnicas utilizadas en estos diferentes valles y aportar de esta manera al conocimiento de las tradiciones técnicas cerámicas del Noroeste Argentino.

Verónica Puente, Mariela Desimone y José Porto presentan un estudio sobre pigmentos y pinturas en Antofagasta de la Sierra en Catamarca, Argentina. A partir del análisis por difracción de rayos $\mathrm{X}$ y microespectroscopía RAMAN caracterizan la composición mineral de las pinturas y 
engobes utilizados para decorar vasijas de diferentes estilos procedentes los sitios arqueológicos de Punta de la Peńa, Peñas Coloradas y Paicuqui, y contribuir de esta manera a nuestra comprensión de las prácticas locales de producción, circulación y consumo de vasijas de cerámica.

El volumen cierra con un artículo de Camila Riera-Soto, Mauricio Uribe, Andrew Menzies y Monserrat Barraza, quienes presentan los resultados de un análisis petrográfico automatizado mediante QEMSCAN de vasijas de cerámica del Formativo Temprano (900 a.C. - 200 d.C.), de la región Tarapacá en el norte de Chile, los cuales confirman la existencia de varios grupos petrográficos que se correlacionan con grupos estilísticos de este período y región, permitiendo acercarnos a la comprensión de la producción y circulación de vasijas de cerámica tanto a nivel local como regional.

En el próximo volumen de este boletín presentaremos los otros nueve artículos que completan esta publicación. Queremos agradecer a los directores del Boletin de Arqueología PUCP, Luis Jaime Castillo y Julián Idilio Santillana, y en especial a su editora, Ana Cecilia Mauricio, sin cuya ayuda esta publicación no hubiese sido posible. 